\title{
A New Design Method for Ultrawideband Microstrip-to-Suspended Stripline Transitions
}

\author{
Young-Gon Kim ${ }^{1}$ and Kang Wook Kim ${ }^{2}$ \\ ${ }^{1}$ LIG Nex1 Co., Ltd. 148-1, Mabuk-dong, Giheung-gu, Yongin-City, Gyeonggi-do, Republic of Korea \\ ${ }^{2}$ School of Electrical Engineering and Computer Science, Kyungpook National University, Sankyuk-dong Buk-gu, \\ Daegu 702-701, Republic of Korea \\ Correspondence should be addressed to Kang Wook Kim; kang_kim@ee.knu.ac.kr
}

Received 23 March 2013; Accepted 4 October 2013

Academic Editor: Tat Yeo

Copyright (c) 2013 Y.-G. Kim and K. W. Kim. This is an open access article distributed under the Creative Commons Attribution License, which permits unrestricted use, distribution, and reproduction in any medium, provided the original work is properly cited.

A clear and efficient design method for ultra-wideband microstrip-to-suspended stripline transition, which is based on the analytical expressions of the whole transitional structure, is presented. The conformal mapping is applied to obtain the characteristic impedance of the transitional structure within $2.85 \%$ accuracy as compared with the EM-simulation results. The transition is designed to provide broadband impedance matching and smooth field conversion. The implemented transition performs less than $0.6 \mathrm{~dB}$ insertion loss per transition for frequencies up to $30 \mathrm{GHz}$.

\section{Introduction}

Among various planar transmission lines used for microwave and millimeter-wave circuits, suspended stripline (SSL) offers some unique advantages as compared with other transmission lines $[1,2]$. (1) SSL yields a higher quality factor than printed stripline and microstrip line (MSL). Since most of the propagated signal energy is carried through the air region rather than through the dielectric layer of the substrate, line losses are considerably reduced, which results in a high Q factor on the order of 500. (2) SSL provides wide impedance ranges because the width of SSL's strip is wider than MSL under the same characteristic impedance. (3) Since the SSL substrate is suspended at the center of a rectangular waveguide, it is easy to design a capacitance overlapped by top and bottom strips, enabling it to implement high-performance ultrawideband filters [2-6]. (4) Due to the shielded structure, radiation leakage is minimized.

On the other hand, MSL is one of the most widely used transmission lines for microwave and millimeter-wave integrated circuits. Using MSL, microwave componentse.g., antennas, couplers, filters, power dividers, and so forthcan be designed with low-cost and compact characteristics [7]. Therefore, to combine the advantages of SSL and MSL simultaneously, it is important to develop a suitable and high performing transition structure between the two different transmission lines, and there were already variously related researches. In [8], an MSL-to-SSL transition using multilayer techniques was reported. The transition bandwidth was up to $12 \mathrm{GHz}$; however, it lacked the ground continuity between the SSL and the microstrip ground plane. In [9], a transition structure between the shielded MSL and SSL was developed with its available bandwidth up to $40 \mathrm{GHz}$. However, a clear design guidance including theoretical analysis was not provided.

In this paper, a new design method of an ultrawideband MSL-to-SSL transition based on theoretically derived closedform equations is proposed. The closed-form equations used to govern the transition structure are obtained by conformal mapping. These equations provide a much easier guidance, helping the SSL-to-MSL transition to reach its optimal performance. The resulted transition has shown a very wideband response with considerably low in-band insertion loss.

\section{Configuration of the Proposed Transition}

The channel cross-section of the suspended stripline is illustrated in Figure 1. In this design, RT/Duroid 4003 with 


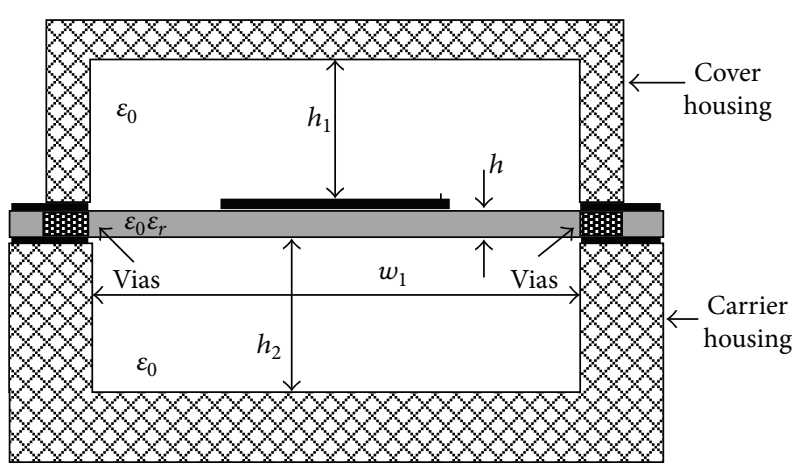

FIGURE 1: Cross-sectional view of the suspended stripline (SSL) channel.

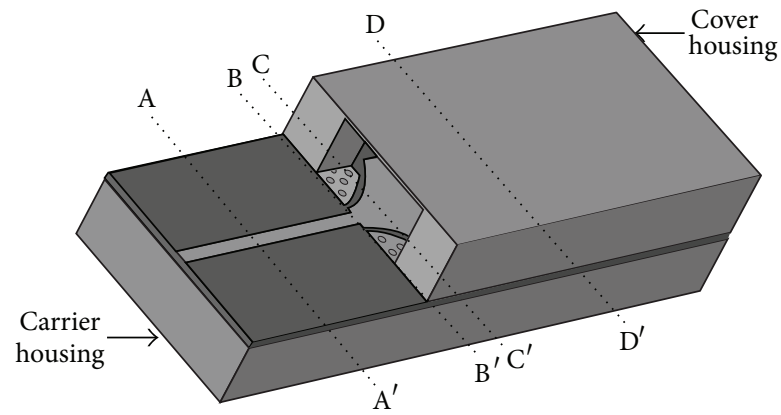

(a)

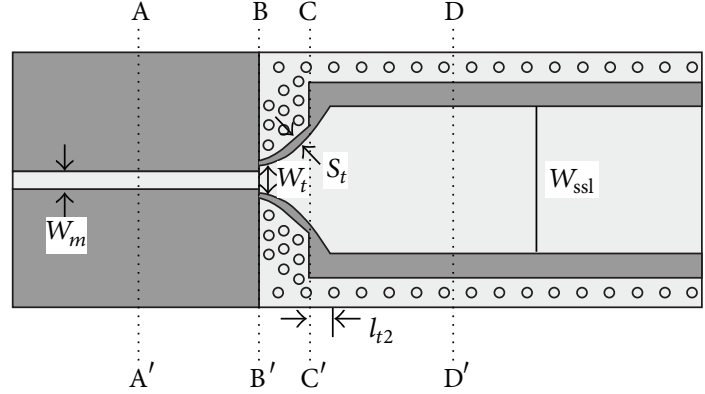

(b)

A $\quad$ B $C$

D

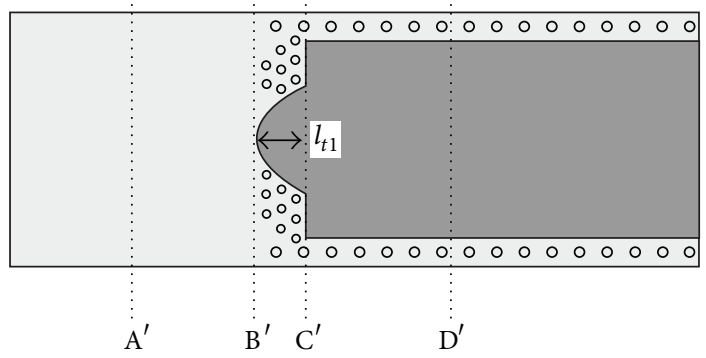

(c)

FIgURE 2: Proposed configuration of the MSL-to-SSL transition: (a) perspective view, (b) top view, and (c) bottom view.

8 mil thickness is used as a circuit substrate $\left(\varepsilon_{r}=3.38\right)$. The channel dimension is carefully chosen to suppress the spurious of waveguide modes. The channel cutoff frequency can be approximately determined by (1) [10]

$$
f_{c}=\frac{c}{2 w_{1}} \sqrt{1-\frac{h}{b}\left(\frac{\varepsilon_{r}-1}{\varepsilon_{r}}\right)}
$$

where $w_{1}$ is the channel width, $b$ is the channel height (= $\left.h_{1}+h_{2}+h\right), c=3 \times 10^{8} \mathrm{~m} / \mathrm{s}$, and $h$ is the substrate height. In this design, in order to operate the circuit up to $30 \mathrm{GHz}$, the channel dimensions, listed in Table 1, are chosen to provide a cutoff frequency at $30 \mathrm{GHz}$. To provide flexible assembly, a split-block waveguide is used; a cover housing can easily be clamped to a bottom carrier housing. The dielectric substrate is suspended on the top of the carrier housing (200 mil width and $80 \mathrm{mil} \mathrm{depth)}$ and is capped with the cover housing (200 mil width and 80 mil height). Multiple vias are placed at the edge of the substrates to provide a good conductive connection between the carrier housing and the cover housing.

Figure 2(a) shows a perspective view of the proposed MSL-to-SSL transition, and Figures 2(b) and 2(c) show top and bottom view of the transition. The transition consists of an MSL section (A- $\mathrm{A}^{\prime}$ ), an MSL-to-SSL transition section (B$\mathrm{B}^{\prime}$ and $\left.\mathrm{C}-\mathrm{C}^{\prime}\right)$, and an SSL section $\left(\mathrm{D}-\mathrm{D}^{\prime}\right)$. Figure 3 displays cross-sectional views of the simplified electric field distributions. In an MSL, electric field lines are perpendicularly terminated to the ground plane of the substrate as shown at $A-A^{\prime}$ in Figure 3. In an SSL, the arrowed lines representing 


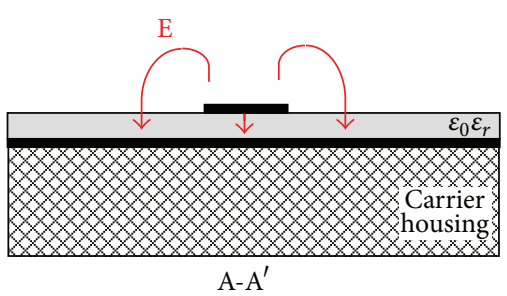

(a)

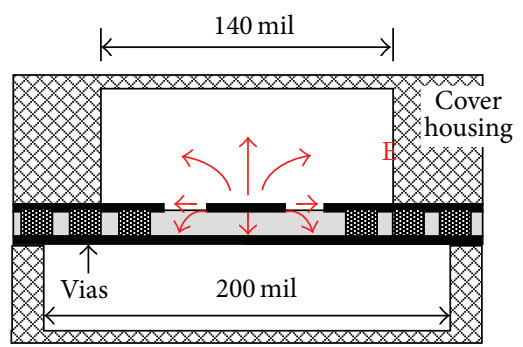

B-B'

(b)

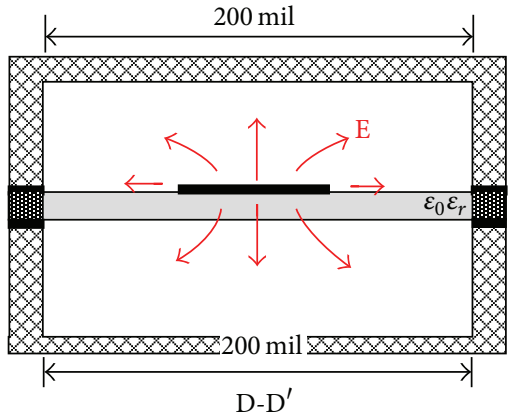

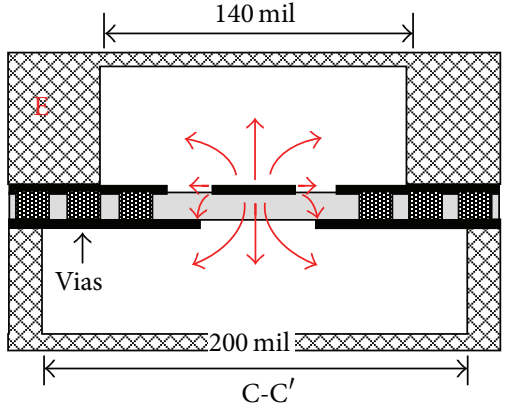

(c)

(d)

Figure 3: Cross-sectional views of simplified electric field distributions.

TABLE 1: Summary of the SSL channel dimensions (unit: mil).

\begin{tabular}{llcc}
\hline$h_{1}$ & $h_{2}$ & $h$ & $w_{1}$ \\
\hline 80 & 80 & 8 & 200 \\
\hline
\end{tabular}

electric flux are conformed to a TEM-like field between the signal line and metal wall as shown at $\mathrm{D}^{-\mathrm{D}^{\prime}}$ in Figure 3. In order to gradually match the field distributions between MSL and SSL, a transitional structure of a shielded conductorbacked coplanar waveguide $\left(\mathrm{B}-\mathrm{B}^{\prime}\right)$ with the cover cavity of 140 mil width and 80 mil height is placed after the MSL section. The electric field lines of the transition structure tend to possess increased horizontal components which are terminated at the edge of the cover (B- $\mathrm{B}^{\prime}$ in Figure 3$)$. As gradually widening width of the aperture on the bottom conductor of the substrate (C-C' in Figure 3 ), the electric flux begins to flow out of the bottom aperture toward to the ground surface of the carrier housing. The width of the top conductor is also changed along the transition to match the characteristic impedance and to provide a smooth field matching to that of SSL. For the transitional structure, the size of the cover cavity is kept as 140 mil width and 80 mil height, and the size of the bottom cavity is maintained as $200 \mathrm{mil}$ width and $80 \mathrm{mil}$ depth. The dimensions of the top and bottom metal strips are varied to provide a smooth transition. In the SSL section, the width of the cover housing becomes 200 mil beyond the transition section (B-B ${ }^{\prime}$ to $\left.C-C^{\prime}\right)$. The dimensions of the proposed transition are summarized in Table 2.
TABLE 2: Summary of the transition dimensions (unit: mil).

\begin{tabular}{llllll}
\hline$w_{m}$ & $w_{t}$ & $w_{\text {ssl }}$ & $s_{t}$ & $l_{t 1}$ & $l_{t 2}$ \\
\hline 17 & 18 & 150 & 5 & 52 & 20 \\
\hline
\end{tabular}

\section{Analysis of the Characteristic Impedance of the Transition}

In designing the transition, impedance matching between adjoining transitional lines is very important. The guideline used for design of the proposed transition is as follows. (1) The shape of the top conductor is determined by the taper suggested in [11]. (2) The shape of the bottom conductor is adjusted to maintain the characteristic impedance of the transitional structure at $50 \Omega$ for broadband matching.

Figure 4 shows the process of determining SSL's top strip layout in the transition region. The diameter of the trace circle lies on the $y$-axis at the beginning of the transition while the $x$-axis is at the center of the signal line. The radius of the trace circle $(r)$ is chosen as $73 \mathrm{mil}$ in this design. Next, the shape of the bottom strip should be decided to maintain a $50 \Omega$ of characteristic impedance along the transition. This paper presents a new method to derive the analytical formula for the shielded CPW transitional structure with an aperture on the bottom side of the substrate (C-C' in Figure 3). The analytical expressions for the $\mathrm{CPW}$ structure with a ground aperture were recently reported in [12]. In this paper, however, new analytical expressions for the shielded CPW structure with a ground aperture using the conformal mapping method are presented. 


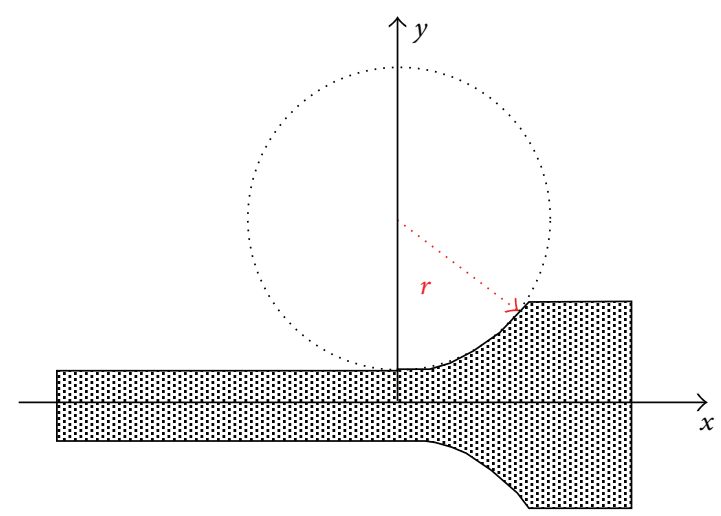

Figure 4: Top plane trace of the transition ( $r=73$ mil).

The configuration of the shielded CPW structure with a ground aperture on the bottom layer is shown in Figure 5(a). In order to obtain the characteristic impedance and effective permittivity of the structure, the Schwarz-Christoffel conformal mapping method is applied. The following assumptions are used for this analysis.

(1) All conductors are perfectly conducting with zero thickness.

(2) The aperture is assumed to be symmetrical with respect to the center of the signal conductor.

(3) The RF signal along the structure propagates as a quasi-TEM mode.

(4) The dielectric boundaries, which can be considered as perfect magnetic walls, exist.

For the analysis, the structure is divided into three regions as shown in Figure 5(b). For each region, the capacitance per unit length has been obtained using the mapping method. The total capacitance per unit length is the sum of capacitances for three regions.

The first transformation to obtain the capacitance in Region I is performed by mapping from $w$-plane to $z$-plane as shown in Figures 6(a) and 6(b) using conformal mapping. Mapping the $w$-plane into $z$-plane is performed by solving

$$
x=\operatorname{sn}\left(\frac{2 K\left(1 / x_{a}\right)}{w_{1}} w, \frac{1}{x_{a}}\right),
$$

where sn is the Jacobi elliptic function and $K$ is the complete elliptic integral. The modulus $1 / x_{a}$ is obtained by

$$
\frac{K\left(1 / x_{a}\right)}{K\left(1 / x_{a}^{\prime}\right)}=\frac{w_{1}}{2 h_{1}}, \quad \frac{1}{x_{a}^{\prime}}=\sqrt{1-\left(\frac{1}{x_{a}}\right)^{2}} .
$$

Points A and B in $w$-plane are transformed to the corresponding points in $z$-plane as

$$
\begin{gathered}
\mathrm{A}: w^{\prime}=\operatorname{sn}\left(\frac{2 K\left(1 / x_{a}\right)}{w_{1}} \frac{w}{2}, \frac{1}{x_{a}}\right), \\
\mathrm{B}: \mathrm{s}^{\prime}=\operatorname{sn}\left(\frac{2 K\left(1 / x_{a}\right)}{w_{1}}\left(\frac{w}{2}+g\right), \frac{1}{x_{a}}\right) .
\end{gathered}
$$

The transformation from Figure 6(b) to 6(c) is the same as obtaining CPW capacitance with air filling [13]. The capacitance is then calculated as

$$
C_{1}=2 \varepsilon_{0} \frac{K\left(k_{0}\right)}{K\left(k_{0}^{\prime}\right)}
$$

The modulus $k_{0}$ and the complementary modulus $k_{0}^{\prime}$ are given by

$$
k_{0}=\frac{w^{\prime}}{s^{\prime}}, \quad k_{0}^{\prime}=\sqrt{1-k_{0}^{2}}
$$

Region II is almost the same structure as Region I except for the existence of an aperture on the bottom plane. Figure 7(a) is transformed into Figure 7(b) by following mapping function:

$$
x=\operatorname{sn}\left(\frac{2 K\left(1 / x_{b}\right)}{w_{2}} w, \frac{1}{x_{b}}\right), \quad \frac{1}{x_{b}^{\prime}}=\sqrt{1-\left(\frac{1}{x_{b}}\right)^{2}},
$$

where the modulus $1 / x_{b}$ is obtained by

$$
\frac{K\left(1 / x_{b}\right)}{K\left(1 / x_{b}^{\prime}\right)}=\frac{w_{2}}{2 h}
$$

Points $\mathrm{A}, \mathrm{B}$, and $\mathrm{E}$ in $w$-plane are transformed to the corresponding points in $z$-plane as

$$
\begin{gathered}
\mathrm{A}: w^{\prime \prime}=\operatorname{sn}\left(\frac{2 K\left(1 / x_{b}\right)}{w_{2}} \frac{w}{2}, \frac{1}{x_{b}}\right), \\
\mathrm{B}: s^{\prime \prime}=\operatorname{sn}\left(\frac{2 K\left(1 / x_{b}\right)}{w_{2}}\left(\frac{w}{2}+g\right), \frac{1}{x_{b}}\right), \\
\mathrm{E}: x_{c}=\operatorname{sn}\left(\frac{2 K\left(1 / x_{b}\right)}{w_{2}}\left(\frac{s}{2}+j h\right), \frac{1}{x_{b}}\right) .
\end{gathered}
$$




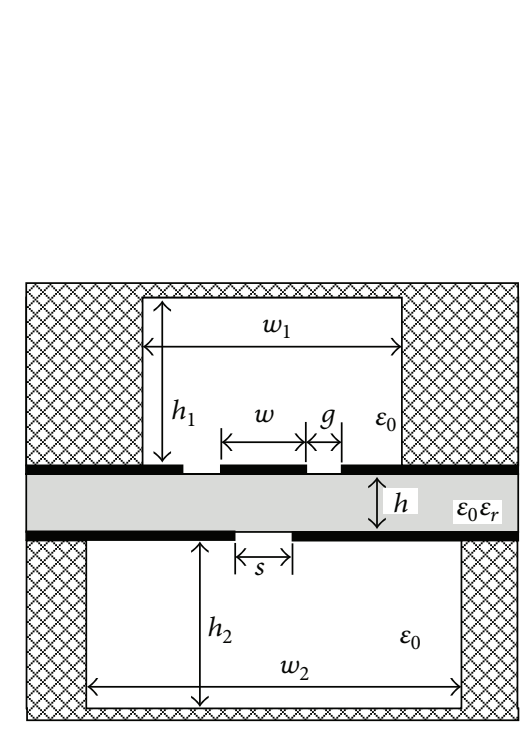

(a)
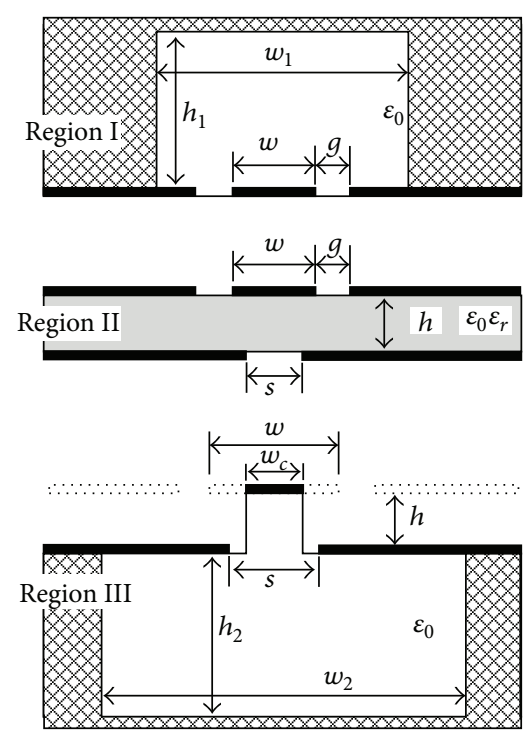

(b)

FIgURE 5: Configuration of the transitional structure: (a) the entire structure and (b) individual structures used in the analysis.

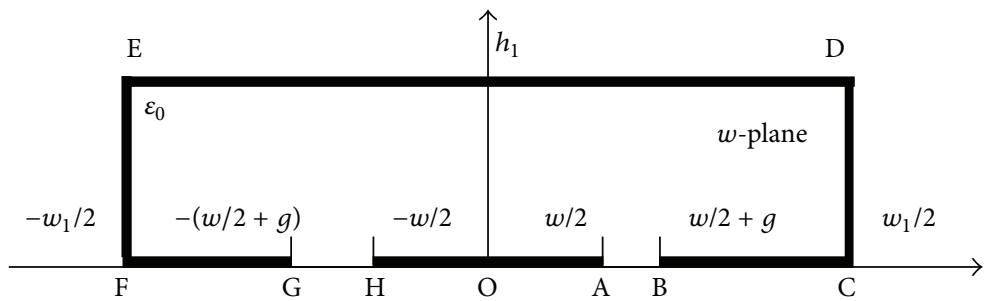

(a)

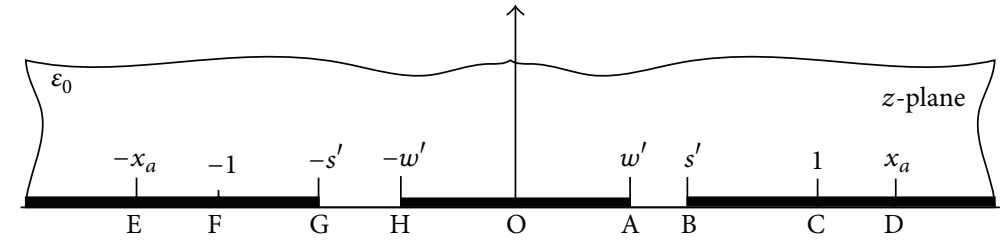

(b)

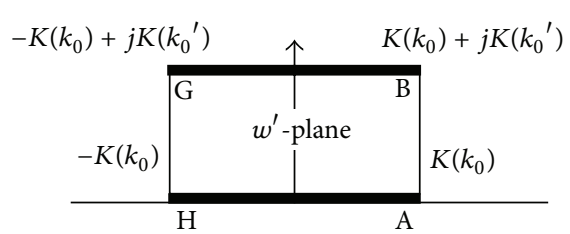

(c)

FIGURE 6: Conformal mapping transformation for Region I of Figure 5(b).

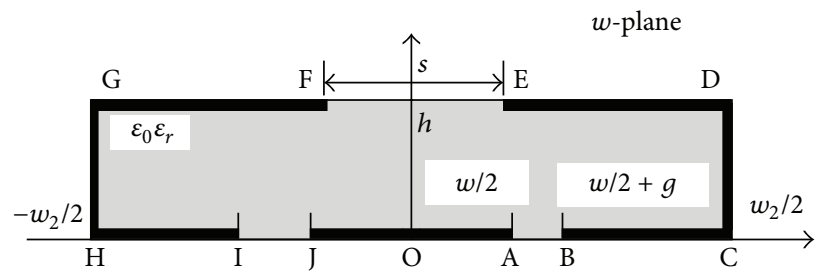

(a)

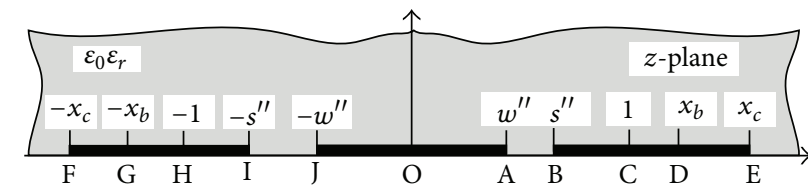

(b)

FIGURE 7: Conformal mapping transformation for Region II. 


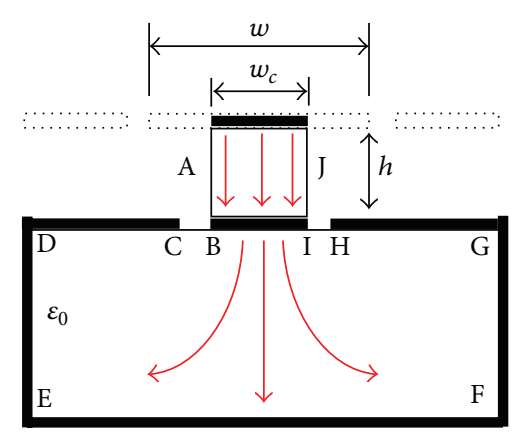

(a)

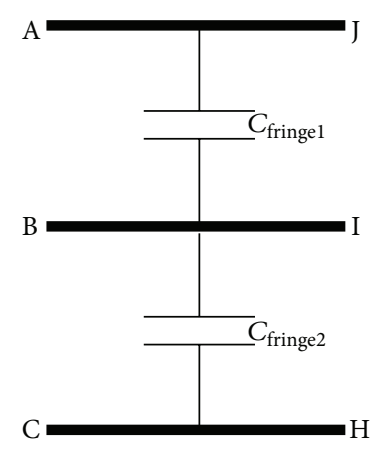

(b)

FIGURE 8: Model of Region III with consideration of leakage field: (a) field distributions and (b) equivalent circuit model.

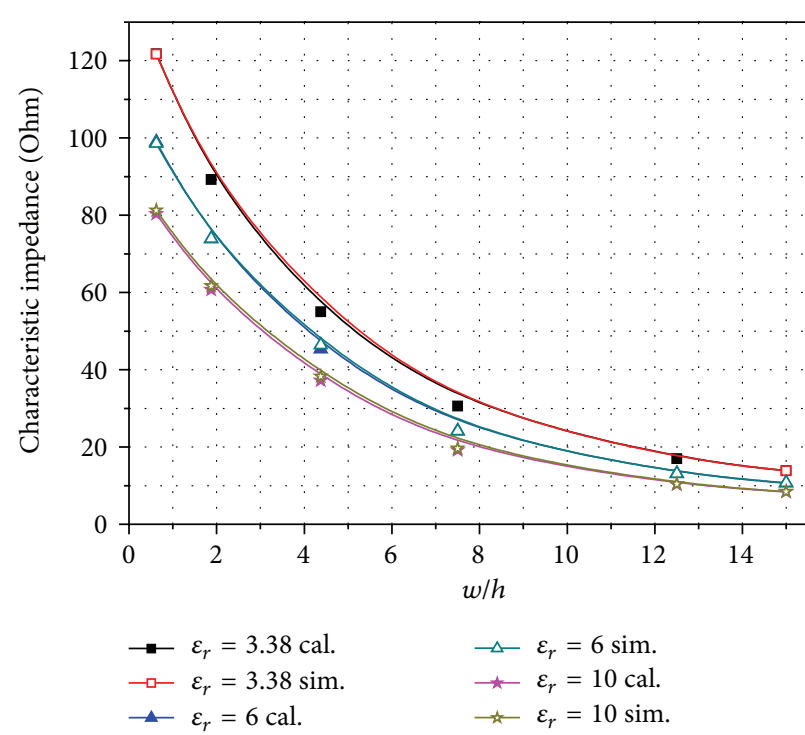

(a)

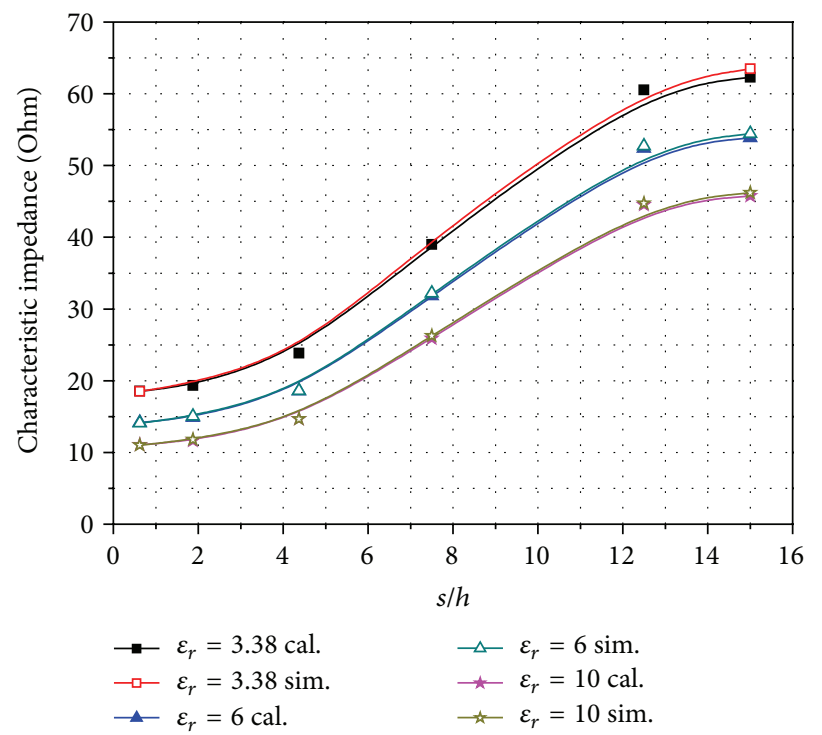

(b)

Figure 9: Characteristic impedances as a function of $w / h$ and $s / h$ (cal.: calculated, sim.: simulated). In case of (a), $s=40 \mathrm{mil}, g=10 \mathrm{mil}$, $h=8 \mathrm{mil}, w_{1}=200 \mathrm{mil}, w_{2}=200 \mathrm{mil}, h_{1}=80 \mathrm{mil}$, and $h_{2}=200 \mathrm{mil}$. In case of (b), $w=70 \mathrm{mil}, g=10 \mathrm{mil}, h=8 \mathrm{mil}, w_{1}=200 \mathrm{mil}$, $w_{2}=200 \mathrm{mil}, h_{1}=80 \mathrm{mil}$, and $h_{2}=200 \mathrm{mil}$.

The transformed structure in Figure $7(\mathrm{~b})$ is the same as the CPW with finite ground planes with air filling in [13]. The capacitance and the modulus are

$$
\begin{gathered}
C_{2}=2 \varepsilon_{0} \varepsilon_{r} \frac{K\left(k_{1}\right)}{K\left(k_{1}^{\prime}\right)}, \\
k_{1}=\frac{w^{\prime \prime}}{s^{\prime \prime}} \sqrt{\frac{1-\left(s^{\prime \prime} / x_{c}\right)^{2}}{1-\left(w^{\prime \prime} / x_{c}\right)^{2}}}, \quad k_{1}^{\prime}=\sqrt{1-k_{1}^{2}} .
\end{gathered}
$$

The shape of Region III is carefully chosen to evaluate the effect of leakage field through the ground aperture on the bottom layer. The amount of leakage field flowing out of the ground aperture on the bottom layer will depend on the aperture width. In order to calculate the capacitance caused by this leakage, the structure for Region III is modeled as Figure 8(a). The signal line width which influences leakage field is changed according to the aperture width $(s)$ and substrate height $(h)$. Points A and J are determined by line of flux departing from Points $\mathrm{C}$ and $\mathrm{H}$, respectively [14]. The signal line width influencing leakage field is determined by the following relations:

$$
\frac{w_{c}}{2}= \begin{cases}\frac{s}{2}-\frac{h}{\pi}, & \text { if } \frac{w}{2}>\frac{s}{2}-\frac{h}{\pi} \\ \frac{w}{2}, & \text { elsewhere. }\end{cases}
$$

Figure 8(a) shows the electric field lines that contribute to capacitance in Region III and the equivalent capacitance model is shown in Figure 8(b), which was similarly suggested 


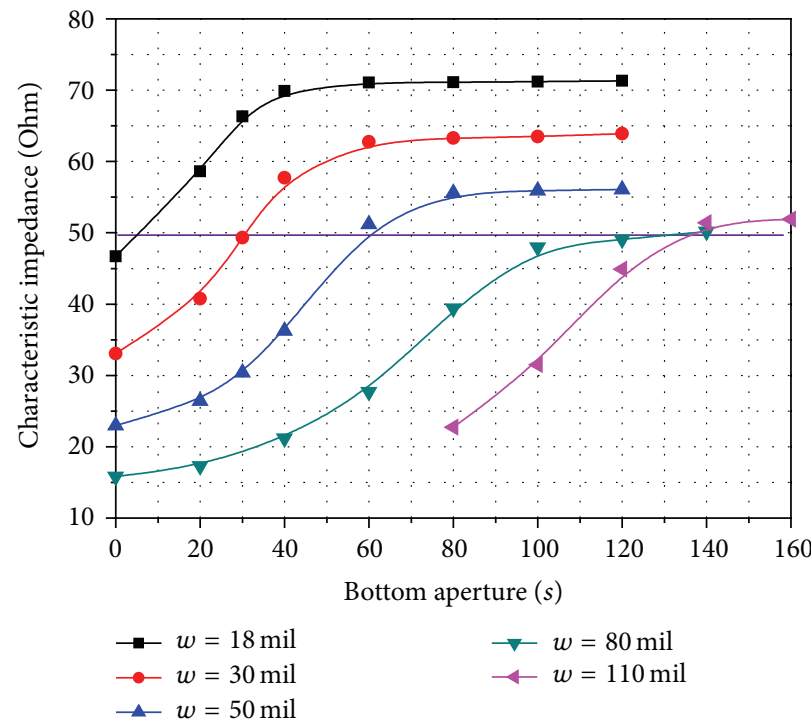

FIGURE 10: Characteristic impedances of the transition to determine the bottom aperture $(s)$.

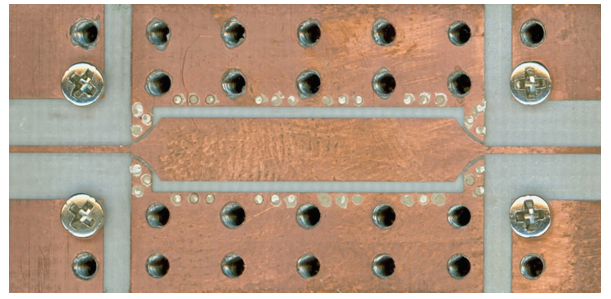

(a)

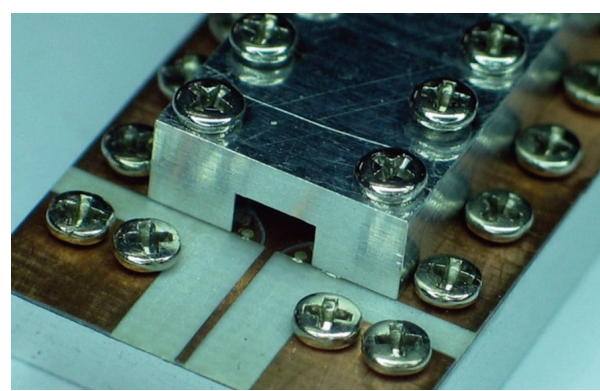

(c)

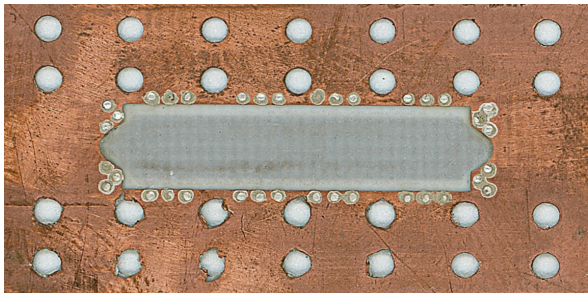

(b)

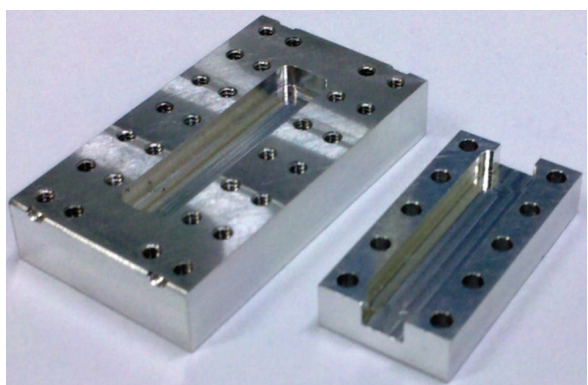

(d)

FIgURE 11: Picture of the fabricated ultrawideband MSL-to-SSL transition in a back-to-back configuration: (a) top view, (b) bottom view, (c) cover and bottom carrier, and (d) perspective view of transition.

in [15]. As shown in Figure 8, the total capacitance influencing Region III is divided into two capacitances $C_{\text {fringel }}$ and $C_{\text {fringe2 }}$ for easy calculation. Capacitance, $C_{\text {fringel }}$ is the parallel plate capacitance between AJ and BI as shown in Figure 8(a) and the capacitance is given by

$$
C_{\text {fringel }}=\varepsilon_{0} \frac{w_{c}}{h} .
$$

Capacitance $C_{\text {fringe2 }}$ is obtained by the same process as the Region I analysis. The final expression for capacitance of Region III is obtained by

$$
C_{3}=\frac{C_{\text {fringe1 }} C_{\text {fringe2 }}}{C_{\text {fringe1 }}+C_{\text {fringe2 }}} .
$$

The characteristic impedance and effective permittivity of the whole structure can be obtained as the sum of capacitance contributions of the three regions. The total capacitance is the sum of (5), (12), and (16). The characteristic impedance is then

$$
Z_{0}=\frac{1}{\sqrt{\varepsilon_{\text {eff }}}} \frac{60 \pi}{\left[K\left(k_{0}\right) / K\left(k_{0}^{\prime}\right)+K\left(k_{1}\right) / K\left(k_{1}^{\prime}\right)+C_{3} / 2\right]} .
$$

The effective dielectric constant can be obtained as the ratio of the total capacitance to the calculated capacitance with air filling instead of the dielectric substrate:

$$
\varepsilon_{\text {eff }}=\frac{K\left(k_{0}\right) / K\left(k_{0}^{\prime}\right)+\varepsilon_{r} K\left(k_{1}\right) / K\left(k_{1}^{\prime}\right)+C_{3} / 2}{K\left(k_{0}\right) / K\left(k_{0}^{\prime}\right)+K\left(k_{1}\right) / K\left(k_{1}^{\prime}\right)+C_{3} / 2} .
$$




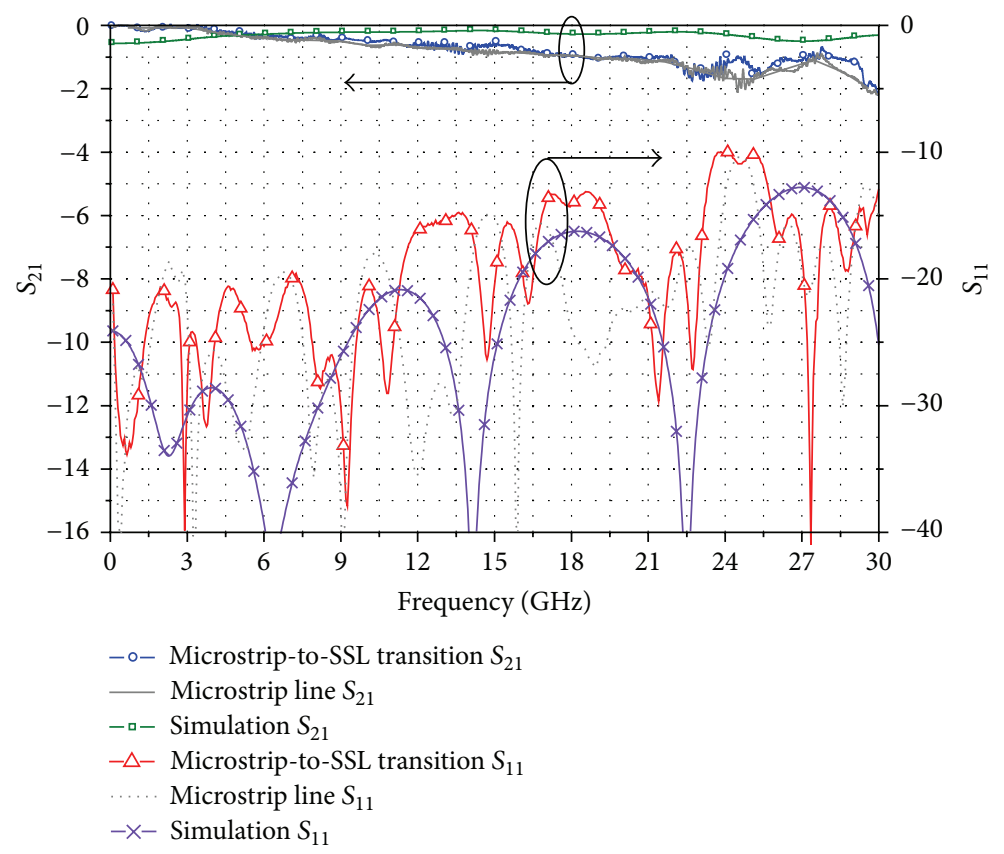

Figure 12: Simulated and measured insertion loss and return loss of the transition.

Figures 9(a) and 9(b) compare the characteristic impedances obtained by the proposed analytical method and the commercial EM simulator (CST Microwave Studio). Figure 9(a) shows characteristic impedance variations with the signal width $(w)$ for various dielectric constants. The impedance values via EM simulation are obtained at $8 \mathrm{GHz}$, but their deviations are within $0.3 \%$ up to $100 \mathrm{GHz}$. Also, the characteristic impedances are obtained with variation of the aperture width $(s)$ for various dielectric constants in Figure 9(b). For both cases, the differences between the analytical expressions and EM simulation results are within $2.85 \%$ (average $1.66 \%$ ).

According to the design process proposed in this paper, the conductor shape of the transition can easily be obtained using the analytical expression of the characteristic impedance (18). The width of the cover cavity for the transition section is chosen as 140 mil to adjust impedance matching. Figure 10 shows computed characteristic impedances for various signal widths $(w)$ as a function of the bottom aperture width $(s)$. The shape of the bottom aperture can be determined by following the $50 \Omega$ line. The top conductor width of the transitional structure is changed from $18 \mathrm{mil}$ to 110 mil with the gap width $(g)$ of 5 mil, the cover width of $140 \mathrm{mil}$, and the transition length $\left(l_{t 1}\right)$ of $52 \mathrm{mil}$. In order to keep the impedance at $50 \Omega$, the width of the bottom aperture is varied from $0 \mathrm{mil}$ to $140 \mathrm{mil}$. When the signal width becomes more than $110 \mathrm{mil}$, the spacing from the edge of the signal line to the top cover is kept at $25 \mathrm{mil}$, and variation of the bottom ground shaping is also stopped.

\section{Simulation and Measurement}

Figure 11 shows the fabricated MSL-to-SSL transition. The size of the back-to-back transition is $1278 \mathrm{mil} \times 700 \mathrm{mil}$.
The performance of the transition was measured using the Anritsu network analyzer 37397C with the transition mounted in an Anritsu universal test fixture. Figure 12 shows the measured and simulated $S$ parameters of the proposed back-to-back transition. Also, for the comparison, the measured results of the MSL with the same length of the proposed transition (1278 mil) are shown. It is observed that the backto-back transition has less than $1.9 \mathrm{~dB}$ insertion loss and more than $10 \mathrm{~dB}$ return loss up to $30 \mathrm{GHz}$. Higher insertion loss at high frequencies $(>18 \mathrm{GHz})$ may have been caused due to fabrication inaccuracy, radiation loss of the MSL, high loss tangent (loss tangent $=0.0027$ ) of the substrate, fabrication tolerances of the SSL housing, and interconnection loss of the test fixture. By subtracting the interconnection losses and the transmission line losses, the insertion loss of the implemented transition is estimated to be less than $0.6 \mathrm{~dB}$ per transition for frequencies up to $30 \mathrm{GHz}$. It is noted that insertion losses of the same-length MSL are higher at frequencies over $23 \mathrm{GHz}$ than those of the proposed transition. The reason of low loss with the SSL transition structure is that the signal propagates mostly through the air with less radiation and dielectric losses as compared with MSL. The estimated insertion loss of the proposed transition as compared with the MSL is less than $0.1 \mathrm{~dB}$ per transition.

\section{Conclusion}

A new clear and efficient design method for the MSL-to-SSL transition based on analytical expressions of the characteristic impedances of the whole transitional structure has been proposed. The differences between the derived analytical expressions and EM simulations are within 2.85\% up to $30 \mathrm{GHz}$. The implemented MSL-to-SSL transition using the design guideline performs ultrawideband with low insertion 
loss and good return loss. The transition design provides broadband impedance matching and smooth field transformation to possess ultrawideband performance. Since this proposed transition can be easily integrated with microstrip based circuits, the proposed transition will find useful applications for circuits simultaneously employing microstrip components and SSL components with ultrawidebandwidth.

\section{Acknowledgments}

This research was supported by National R\&D Program through the National Research Foundation of Korea (NRF) funded by the Ministry of Education, Science and Technology (NRF-2012M1A7A1A02034753).

\section{References}

[1] L. G. Maloratsky, "Reviewing the basics of suspended striplines," Microwave Journal, vol. 45, no. 10, pp. 82-98, 2002.

[2] M.-H. Ho and P.-F. Chen, "Suspended substrate stripline bandpass filters with source-load coupling structure using lumped and full-wave mixed approach," Progress in Electromagnetics Research, vol. 122, pp. 519-535, 2012.

[3] X.-J. Liao, M.-H. Ho, W.-H. Hsu, W.-Q. Xu, and L.-J. Lin, "Quasi-lumped design of UWB BPF using suspended stripline," Progress in Electromagnetics Research Letters, vol. 11, pp. 65-72, 2009.

[4] M. Chen, C.-Y. Jiang, W.-Q. Xu, and M.-H. Ho, "Design of high order suspended stripline bandpass filter with miniaturization," Progress in Electromagnetics Research Letters, vol. 8, pp. 9-17, 2009.

[5] W. Schwa, F. Bogelsack, and W. Menzel, "Multilayer suspended stripline and coplanar line filters," IEEE Transactions on Microwave Theory and Techniques, vol. 46, no. 7, pp. 1403-1407, 1994.

[6] J. D. Rhodes, "Suspended substrate filters and multiplexers," in Proceedings of the 16th European Microwave Conference, pp. 816, 1986.

[7] K. C. Gupta, R. Garg, I. Bahl, and P. Bhartia, Microstrip Lines and Slotlines, Artech House, Norwood, Mass, USA, 1996.

[8] W. Schwab and W. Menzel, "Suspended stripline to microstrip transition using multilayer techniques," in Proceedings of the 22nd European Microwave Conference, vol. 2, pp. 1181-1186, August 1992.

[9] S. Lin and M. Eron, "Development of an ultra-wideband suspended stripline to shielded microstrip transition," IEEE Microwave and Wireless Components Letters, vol. 21, no. 9, pp. 474-476, 2011.

[10] H. O. Roy, S. Mandal, A. K. Shukla, and A. K. Kush, "Waveguide to suspended stripline transition techniques at $140 \mathrm{GHz}$," in Proceedings of the International Conference of Recent Advances in Microwave Theory and Applications (MICROWAVE '08), pp. 110-112, November 2008.

[11] A. Beyer and I. Wolff, "Finline taper design made easy," International Microwave Symposium Digest, vol. 22, no. 3, pp. 144-147, 1976.

[12] Y.-G. Kim and K. Kim, "Analysis of coplanar waveguide with a bottom ground aperture," IEEE Microwave and Wireless Components Letters, vol. 22, no. 11, pp. 550-552, 2012.
[13] G. Ghione and C. U. Naldi, "Coplanar waveguides for MMIC applications: effect of upper shielding, conductor backing, finite-extent ground planes, and line-to-line coupling," IEEE Transactions on Microwave Theory and Techniques, vol. 35, no. 3, pp. 260-267, 1987.

[14] N. Pesonen, W. K. Kahn, R. A. Allen, M. W. Cresswell, and M. E. Zaghloul, "Application of conformal mapping approximation techniques: parallel conductors of finite dimensions," IEEE Transactions on Instrumentation and Measurement, vol. 53, no. 3, pp. 812-821, 2004.

[15] A. Bansal, B. C. Paul, and K. Roy, "An analytical fringe capacitance model for interconnects using conformal mapping," IEEE Transactions on Computer-Aided Design of Integrated Circuits and Systems, vol. 25, no. 12, pp. 2765-2774, 2006. 

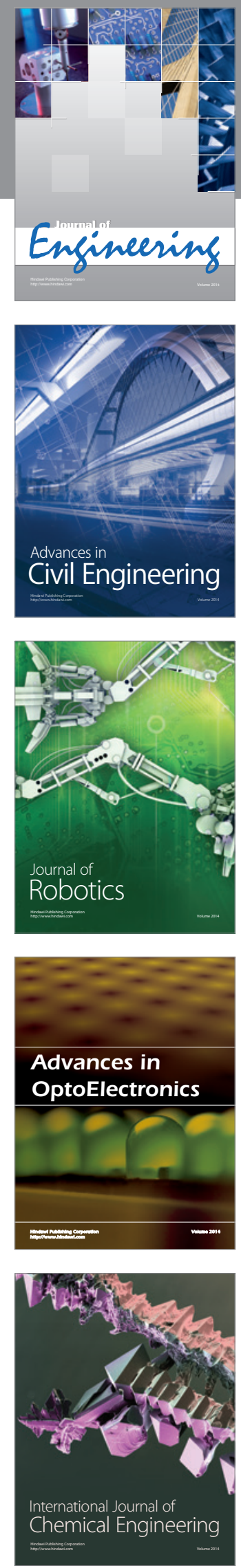

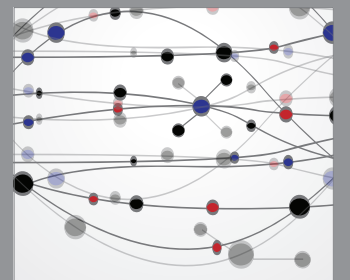

The Scientific World Journal
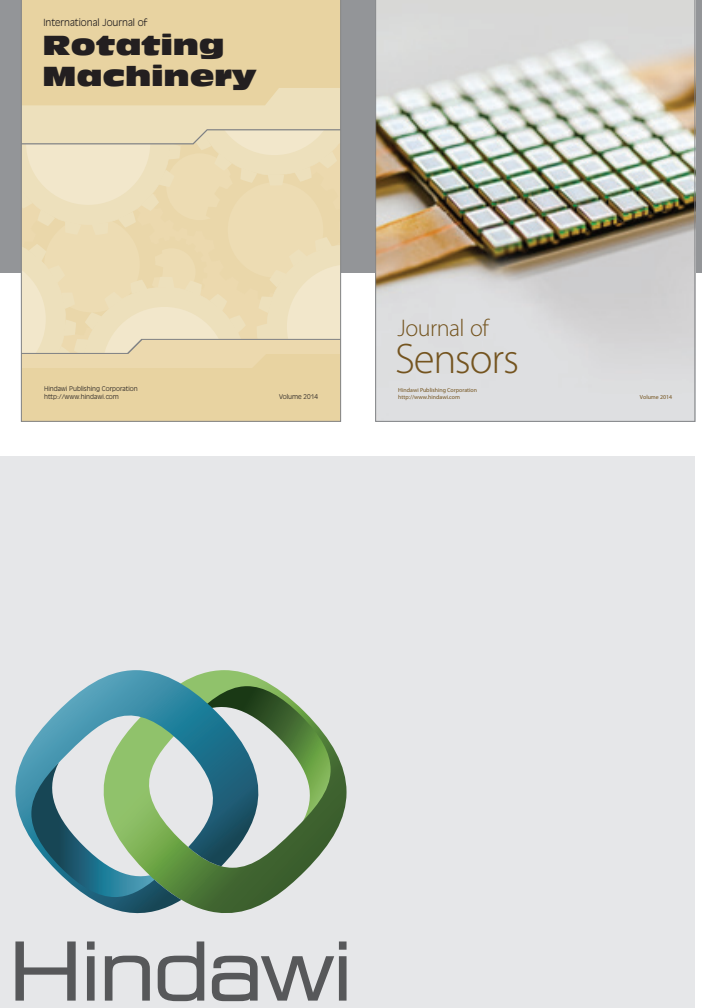

Submit your manuscripts at http://www.hindawi.com
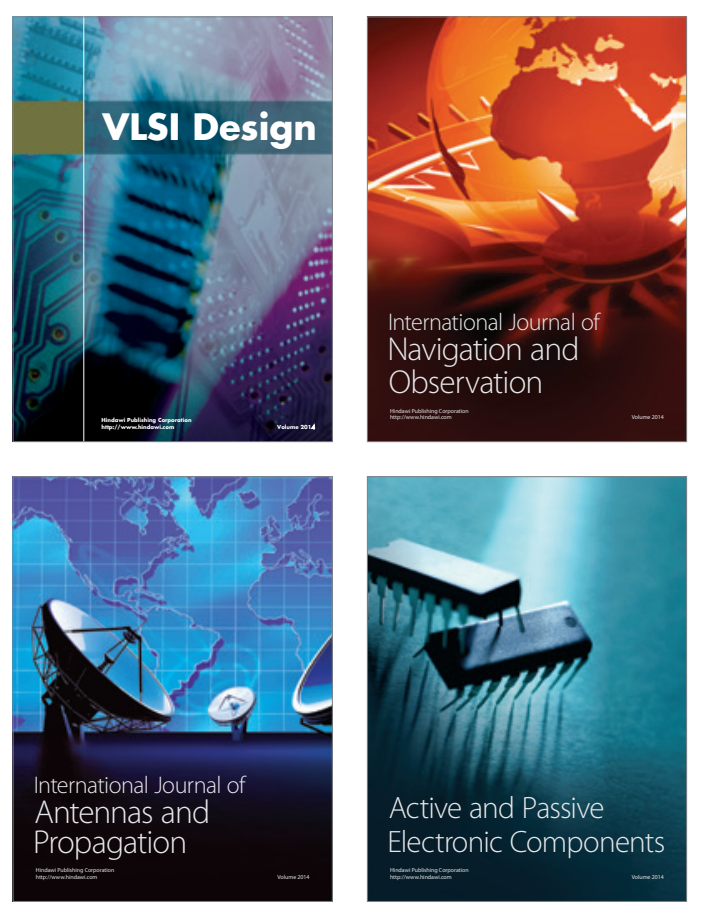
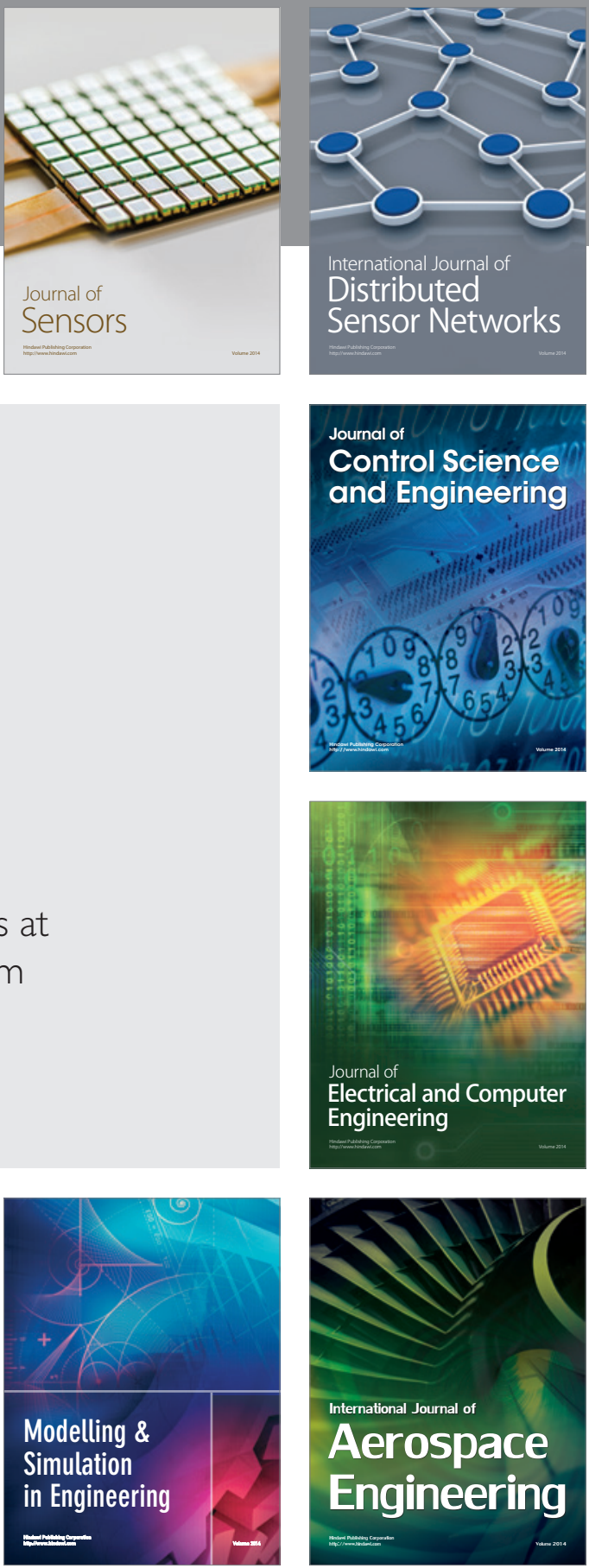

Journal of

Control Science

and Engineering
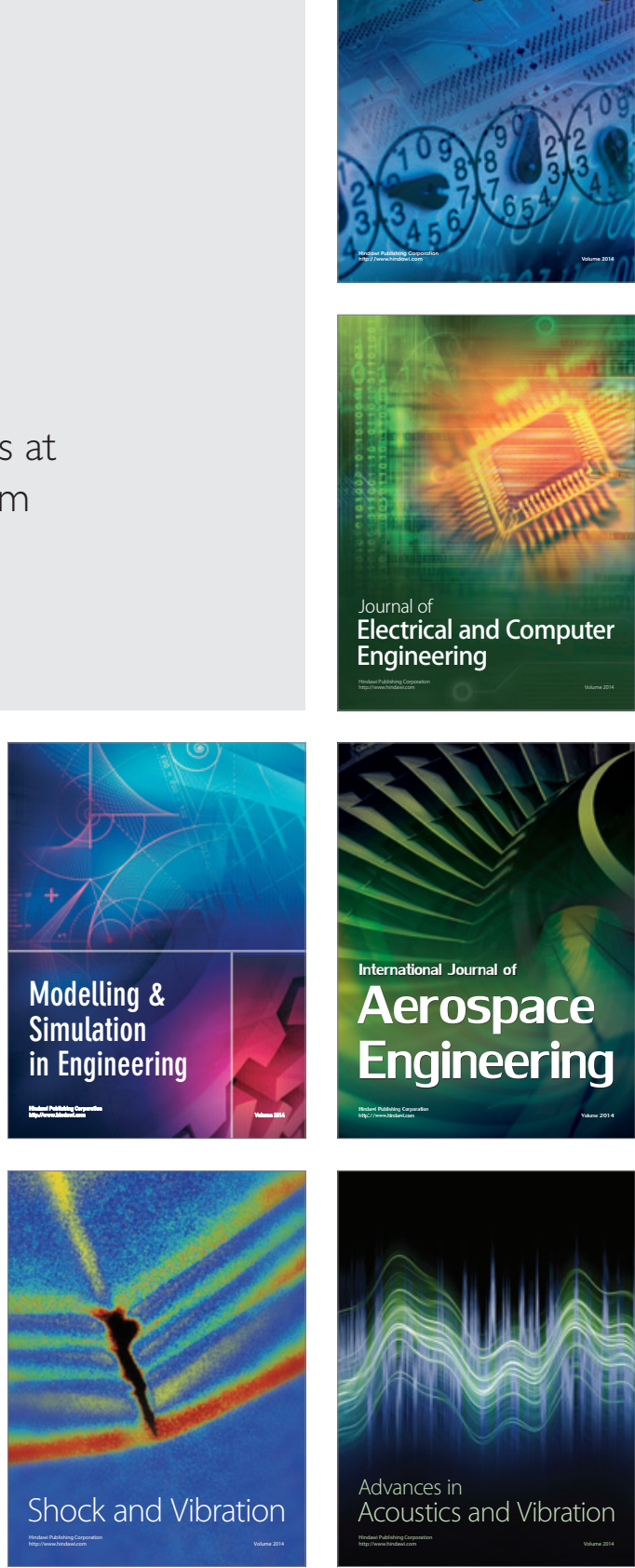